\title{
Interação medicamentosa em usuários de antidepressivos do sistema público de um município do sul do Brasil
}

\section{Drug interaction in patients to public health system in antidepressants use in Southem of Brazil}

\author{
Maiara Schenkela, Juliana Simão ${ }^{b}$, Karin Hepp Schwanbachc ${ }^{c}$ Christiane de Fátima Colet ${ }^{d}$ \\ a Farmacêutica graduada pela Universidade Regional do Noroeste do Estado do Rio Grande do Sul (UNIJUÍ). \\ b Acadêmica do Curso de Graduação em Farmácia da UNIJUÍ. \\ c Farmacêutica. Prefeitura Municipal de Porto Alegre. \\ d Farmacêutica. Professora do Departamento de Ciências da Vida da UNIJUí.
}

RESUMO

Introdução: A depressão é um problema de saúde pública cujo tratamento se faz com uso de antidepressivos, os quais devem ser usados com cautela, devido aos seus efeitos colaterais e potenciais de interações medicamentosas. Objetivo: Identificar e classificar as principais interações medicamentosas em potencial de antidepressivos com outros medicamentos prescritos.

Materiais e Métodos: Foi realizado um estudo com usuários de antidepressivos, que retiraram estes medicamentos na Unidade Básica de Saúde do município de São José do Inhacorá/RS. Os entrevistados foram localizados a partir de uma consulta ao banco de dados, realizada nos meses de dezembro de 2013 a janeiro de 2014. As entrevistas foram realizadas nas residências com auxilio de um instrumento de coleta de dados elaborado pelos autores e validado por estudo piloto. As interações medicamentosas potenciais foram pesquisadas na base de dados Micromedex ${ }^{\circledR}$ e aqueles medicamentos não encontrados foram pesquisados em livros sobre interação medicamentosa, e foram classificadas pela gravidade em maior, moderada e menor.

Resultados: Foram entrevistados 88 pacientes, com idade média de 56,68 $\pm 16,36$. Foram encontradas 127 interações medicamentosas em potencial, sendo que 42 (47,72\%) estavam sujeitos a interações, com média de 5,90 $\pm 2,73$ por usuário. Quanto à gravidade, 69 (54,33\%) eram maior, 53 (41,73\%) moderada e 5 (3,94\%) menor.

Conclusão: Este estudo demonstra a ocorrência das interações medicamentosas em potencial em grande parte dos usuários, bem como a prática de polifarmácia, que representa um risco ao usuário. Por isso, verifica-se a importância do farmacêutico inserido na equipe multidisciplinar, para poder minimizar os erros relacionados à farmacologia e melhorar a qualidade de vida dos pacientes.

Palavras-chave: depressão; antidepressivos; conduta do tratamento medicamentoso. be used with caution due to its side effects and potential drug interactions.

Objective: To identify and classify the main potential drug interactions between antidepressants and other drugs.

Materials and methods: The research was conducted with patients that use antidepressants and received it from the Basic Health Unity of the city of São José do Inhacorá, RS, Brazil. The subjects were located by searching the database of appointments that took place from December 2013 to January 2014. The interviews were conducted at their homes by using a data collection instrument developed by the authors and validated by a pilot study. Potential drug interactions were researched in the Micromedex ${ }^{\circledR}$ database. The missing interactions on that database were further investigated on books regarding drug interactions and were classified for risk assessment as being of high, moderate and low importance.

Results: A total of 88 patients were interviewed, the mean age was $56.68 \pm 16.36$ years old. A total of 127 potential drug interactions were detected and $42(47.72 \%)$ patients were directly subjected to it, with a mean of $5.90 \pm 2.73$ per patient. Regarding the risk assessment, the interactions were classified as high in 69 (54.33\%), moderate in 53 (41.73\%) and low in 5 (3.94\%).

Conclusion: This study demonstrates the occurrence of potential medical interactions in a significant portion of the patients, as well as the self-medication practice, which also represents a risk for the users. Therefore, it is important that the pharmacist is part of the multidisciplinary team, aiming to minimize the pharmacological related errors and to improve the quality of life of the patients.

Keywords: depression; antidepressants; conduct drug treatment. 


\section{INTRODUÇÃO}

Os transtornos do humor compreendem um grande grupo de transtornos em que o humor patológico e perturbações associadas dominam o quadro clínico. Podem ser considerados como síndromes, pois apresentam um conjunto de sinais e sintomas persistentes por semanas ou meses, com desvio do desempenho habitual do indivíduo e que tendem a recorrer de forma periódica ou cíclica. Existe a perda do controle dos estados de humor e afetos, levando a uma experiência subjetiva de grande sofrimento e frequentemente ocorre um comprometimento do desempenho interpessoal, social e ocupacional. Os episódios depressivos caracterizam o transtorno depressivo maior (TDM) ${ }^{1}$.

O TDM tem prevalência durante a vida de cerca de $15 \%{ }^{1}$, sendo mais comum entre mulheres ${ }^{2}$. A incidência é de $15 \%$ em atenção primária, e o mesmo valor entre pessoas hospitalizadas. Existem vários fatores relacionados na etiologia da doença, como: biológicos (desregulação de neurotransmissores, causas neuroendócrinas, alterações do ritmo circadiano), fatores genéticos e fatores psicossociais (acontecimentos na vida e estresse ambiental, fatores de personalidade). A ansiedade afeta $90 \%$ dos pacientes deprimidos. Além disso, pode estar associada com a dependência de álcool ou outras drogas ${ }^{1}$. Mesmo que a incidência da depressão seja elevada estima-se que muitas vezes esta doença possa ser subdiagnosticada e subtratada $^{2}$, principalmente pela presença de sintomas que também podem ocorrer em doenças crônicas, como fadiga e anorexia. A associação entre depressão e doenças clínicas é muito frequente, levando a pior evolução, tanto do quadro psiquiátrico como da doença clínica, com menor adesão às orientações terapêuticas, além de maior morbidade e mortalidade. Destaca-se que outras doenças estão claramente associadas à depressão, especialmente as cardiovasculares, endocrinológicas, neurológicas, renais, oncológicas e outras síndromes dolorosas crônicas ${ }^{3}$.

O tratamento dos transtornos de humor, como a depressão, visa em primeiro lugar à segurança do paciente, busca avaliação diagnóstica completa e tratamento não só sintomático, mas buscando o bem-estar futuro do paciente ${ }^{4}$. Segundo a Organização Mundial de Saúde ${ }^{4}$, a terapia psicossocial associada com o tratamento farmacológico apresenta os melhores resultados, e o acesso aos psicofármacos essenciais, juntamente com o atendimento por pessoal capacitado, são essenciais para um tratamento eficaz. Os psicofármacos essenciais servem para aliviar os sintomas, abreviar o curso das enfermidades, reduzir a incapacidade e prevenir recaídas. Os antidepressivos são efetivos no tratamento agudo das depressões moderadas e graves, para melhorar os sintomas (resposta) ou eliminalos (remissão completa). Há evidência na literatura de que a permanência de sintomas residuais de depressão está associado a pior qualidade de vida, pior funcionalidade, maior risco de suicídio, maior risco de recaída e aumento de consumo de serviços de saúde. Os tratamentos psicológicos específicos para episódio depressivo são efetivos com maiores evidências para depressões leves a moderadas ${ }^{2}$.

O tratamento farmacológico do TDM utiliza várias classes de medicamentos, sendo que os antidepressivos comumente disponíveis têm eficácia comparável. Os inibidores seletivos da recaptação da serotonina (ISRS) apresentam menos efeitos colaterais, o que pode refletir em um menor índice de abandono do tratamento ${ }^{2}$ e suas meias-vidas prolongadas permitiam administração em dose única diária ${ }^{5}$. O tratamento farmacológico teve início na segunda metade da década de 1950, com o desenvolvimento dos antidepressivos tricíclicos (ADT) e posteriormente os inibidores da monoaminaoxidade $(\mathrm{IMAO})^{5}$. Apesar de os antidepressivos mostrarem-se eficazes e seguros, deve-se ter cautela quanto ao uso destes medicamentos, pois esses fármacos podem causar efeitos colaterais em diversos sistemas orgânicos. Alguns ADTs como amitriptilina, podem causar sonolência e sedação exagerada, e com frequência confusão mental motivada por doses excessivas. O uso dos ISRS está relacionado a distúrbios gastrointestinais, cefaleia, agitação, pânico, insônia e disfunções sexuais, além de sintomas extrapiramidais como distonia, ansiedade e tremores ${ }^{2}$.

Além dos efeitos adversos a que usuários de antidepressivos estão suscetíveis, estes pacientes geralmente são polimedicados, ou seja, usam vários medicamentos simultaneamente, estando mais sujeitos a interações medicamentosas ${ }^{6}$. A interação medicamentosa é uma das variáveis que afeta o resultado terapêutico e quanto maior o número de medicamentos que o paciente faz uso, maior a probabilidade de ocorrência de interações. Estima-se que para usuários de 2 a 3 medicamentos o percentual seja de 3 a $5 \%$, nos que utilizam de 10 a medicamentos, eleva-se para $20 \%$. Estes dados foram observados em um estudo realizado em uma farmácia magistral da cidade de Cascavel/PR que visou detectar as interações entre fármacos antidepressivos e outros associados, com 151 pacientes, sendo que 24 apresentaram potencial para o desenvolvimento de interações medicamentosas em diferentes graus de severidade ${ }^{8}$.

Apesar das interações medicamentosas serem um dos tópicos mais importantes da farmacologia para a prática clínica dos profissionais da saúde, a frequência das interações clinicamente importantes é pouco descrita na literatura ${ }^{9}$. Dessa forma é de suma importância que sejam desenvolvidos estudos que discutam as interações medicamentosas ao qual 
estão suscetíveis os pacientes em uso de medicamentos antidepressivos, que possam piorar o quadro clínico destes pacientes, com consequências sobre sua qualidade de vida. Portanto, esse estudo tem com objetivo identificar e classificar as principais interações medicamentosas em potencial de antidepressivos com outros medicamentos prescritos na atenção primária à saúde.

\section{MATERIAIS E MÉTODOS}

Foi desenvolvido um estudo transversal, de caráter descritivo e analítico. A pesquisa foi realizada com usuários de antidepressivos, que retiraram estes medicamentos na Unidade Básica de Saúde (UBS) do município de São José do Inhacorá/RS. Os entrevistados foram localizados a partir de uma consulta ao banco de dados, realizada nos meses de dezembro de 2013 a janeiro de 2014. O projeto foi aprovado pelo Comitê de Ética em Pesquisa da UNIJUí (CEP/ UNIJUÍ) no 508.920/2013.

Foram incluídos no estudo todos os usuários, maiores de 18 anos, que utilizam medicamentos antidepressivos dispensados pela farmácia da UBS no período supracitado. Foram excluídos do estudo aqueles que não se encontraram na residência no horário das visitas, que suspenderam o tratamento, os que não tinham condições de responder e que não residiam mais no município.

Para a coleta de dados foi utilizado um questionário semiestruturado, com perguntas relacionadas a características socioeconômica e uso de medicamentos antidepressivos. O instrumento de coleta de dados foi validado por meio de estudo piloto. As entrevistas foram realizadas nas residências dos pacientes entre os meses de janeiro a julho de 2014.

As interações medicamentosas potenciais foram pesquisadas na base de dados Micromedex ${ }^{\circledR}$ e aqueles medicamentos não encontrados foram pesquisados em livros sobre interação medicamentosa ${ }^{10,11}$. As interações foram classificadas em maior, moderada e menor. As com gravidade maior foram consideradas aquelas que podiam resultar em risco à vida do paciente e/ou requerer intervenção medicamentosa para minimizar ou prevenir sérios efeitos adversos; moderadas foram aquelas que podiam resultar em exacerbação da condição do paciente e/ou requerer uma alteração na terapia medicamentosa e menores interações com efeitos clínicos limitados, podendo as manifestações incluir um aumento na frequência ou gravidade dos efeitos adversos, mas geralmente não requerendo uma alteração maior na terapia.

Os dados obtidos foram analisados por meio do software Statistical Packager for the Social Sciences (SPSS) (versão 18.0), sendo realizada análise descritiva simples e estatística analítica. As análises descritivas foram apresentadas através de média e desvio padrão para dados contínuos e valores absolutos/relativos (frequência e percentual) para dados categóricos. Além disso, foi aplicado o teste de qui-quadrado para verificação de possíveis associações. Os resultados foram considerados significativos quando $p<0,05$.

\section{RESULTADOS}

Foram entrevistados 88 pacientes, com idade média de $56,68 \pm 16,36$, sendo que a idade mínima foi de 21 anos e máxima de 89 anos e n (75\%) dos usuários eram do sexo feminino. Houve uma média de 4,03 $\pm 2,70$ fármacos por paciente, sendo que $16(18,18 \%)$ faziam uso apenas do antidepressivo, não relatando uso de outros fármacos. Os antidepressivos mais prescritos foram sertralina $\mathrm{n}(28 \%)$, seguido pela fluoxetina $\mathrm{n}(26 \%)$.

Foram encontradas 127 interações medicamentosas em potencial. Dentre os entrevistados, $30(34,1 \%)$ faziam uso de mais que um medicamento, sem nenhuma interação potencial identificada. Já $42(47,72 \%)$ usuários estavam sujeitos a interações, com média de 3,02 $\pm 2,73$ interações por usuário. As interações, classificadas de acordo a gravidade, estão apresentadas na Tabela 1 e observa-se que houve destaque para as interações maiores que representaram n $(54,33 \%)$ das interações descritas. As interações maiores mais prevalentes encontradas foram: fluoxetina/amiodarona, fluoxetina/imipramina e fluoxetina/ amitriptilina, destaca-se que as últimas duas ocorrem pela associação de dois antidepressivos. A Tabela 2 mostra as interações mais frequentes encontradas.

Entre os antidepressivos utilizados pelos entrevistados o grupo mais prevalente foi inibidores seletivos da recaptação da serotonina (ISRS) com n $(63,91 \%)$, com destaque para os fármacos sertralina e fluoxetina, seguidos pelos inibidores da monoaminoxidase (IMAO não seletivo) com n (27,03\%). Destaca-se que as interações entre fármacos antidepressivos ISRS com outros fármacos utilizados pelos entrevistados ocorreram em $26(21,31 \%)$ usuários, sendo que destas, 27 $(72,98 \%)$ eram de gravidade maior, $9(24,32 \%)$ moderadas e apenas $1(2,70 \%)$ de gravidade menor.

Foram prescritos 12 antidepressivos distintos, sendo o citalopram o antidepressivo mais envolvido nas interações, com n (40,54\%). Verificou-se que em 9 usuários, as interações em potencial estavam relacionadas com a prescrição simultânea de antidepressivos de grupos distintos, ISRS e IMAO não seletivos, com destaque para: sertralina $\mathrm{x}$ citalopram ou imipramina ou nortriptilina; fluoxetina $x$ amitriptilina ou imipramina; citalopram $x$ imipramina; amitriptilina $x$ venlafaxina ou citalopram; trazodona $x$ amitriptilina. Dentre as interações observadas entre antidepressivos citados acima, a maioria foi classificada como de gravidade maior. A Tabela 3 mostra todos os antidepressivos envolvidos em interações. 
A prevalência de interações associou-se fortemente com o número de medicamentos prescritos. Foi realizado o teste de associação de qui-quadrado para amostras independentes e observou-se que houve associação entre o número de medicamentos e as chances de interação medicamentosa sendo que quanto mais medicamentos utilizados mais interações medicamentosas em potenciais foram verificadas $(p<0,001)$.

Tabela 1. Classificação das interações medicamentosas quanto à gravidade entre usuários de antidepressivos de um município do interior do Estado do Rio Grande do Sul $(\mathrm{n}=88)$.

\begin{tabular}{|c|c|c|}
\hline & Gravidade & Frequência $\mathbf{n}(\%)$ \\
\hline \multirow[t]{3}{*}{ Interações medicamentosas } & Maior & $69(54,33)$ \\
\hline & Moderada & $53(41,73)$ \\
\hline & Menor & $5(3,94)$ \\
\hline Total & & 127 (100) \\
\hline
\end{tabular}

Tabela 2. Interações medicamentosas gerais potenciais maiores e moderadas mais frequente entre usuários de antidepressivos de município do interior do Estado do Rio Grande do Sul ( $n=88$ ).

\begin{tabular}{|c|c|c|c|}
\hline \multicolumn{3}{|c|}{ Interações Medicamentosas Potenciais } & n (\%) \\
\hline \multirow[t]{6}{*}{ Maiores } & Fluoxetina & $\begin{array}{l}\text { Amiodarona } \\
\text { Imipramina } \\
\text { Amitriptilina }\end{array}$ & $\begin{array}{l}3(3,89) \\
3(3,89) \\
3(3,89)\end{array}$ \\
\hline & Nortriptilina & Sertralina & $3(3,89)$ \\
\hline & Imipramina & $\begin{array}{l}\text { Amiodarona } \\
\text { Resperidona }\end{array}$ & $\begin{array}{l}2(2,59) \\
2(2,59)\end{array}$ \\
\hline & Amiodarona & $\begin{array}{l}\text { Sotalol } \\
\text { Digoxina }\end{array}$ & $\begin{array}{l}2(2,59) \\
2(2,59)\end{array}$ \\
\hline & Sotalol & Furosemida & $2(2,59)$ \\
\hline & & Outros & $55(71,49)$ \\
\hline \multirow[t]{4}{*}{ Moderadas } & Enalapril & $\begin{array}{l}\text { Hidroclorotiazida } \\
\text { Furosemida }\end{array}$ & $\begin{array}{l}4(7,69) \\
4(7,69)\end{array}$ \\
\hline & Fluoxetina & Levotiroxina & $3(5,76)$ \\
\hline & Sotalol & Digoxina & $2(3,84)$ \\
\hline & & Outros & $39(75,02)$ \\
\hline
\end{tabular}

Tabela 3. Descrição das interações medicamentosas de antidepressivos com outros medicamentos, gravidade e risco potencial de usuários de antidepressivos do município do interior do Estado do Rio Grande do Sul ( $\mathrm{n}=88$ ).

\begin{tabular}{|c|c|c|c|c|c|}
\hline Grupo Farmacológico & Antidepressivo & Associado & Risco Potencial & Gravidade & $n(\%)$ \\
\hline \multirow[t]{4}{*}{ ISRS } & \multirow[t]{4}{*}{ Sertralina } & *Citalopram & $\begin{array}{l}\text { Pode resultar em aumento do risco de } \\
\text { síndrome da serotonina }\end{array}$ & & $4(4,46)$ \\
\hline & & *Imipramina & $\begin{array}{l}\text { Resultar em elevações modestas nos níveis séricos } \\
\text { de imipramina ou síndrome possível serotonina }\end{array}$ & & $2(2,23)$ \\
\hline & & Diazepam & Discreto aumento da concentração de diazepam & & $2(2,23)$ \\
\hline & & *Nortriptilia & $\begin{array}{l}\text { Resultar em níveis séricos de nortriptilina elevadas } \\
\text { ou possível Síndrome de serotonina }\end{array}$ & & $1(0,74)$ \\
\hline \multirow[t]{5}{*}{ ISRS } & \multirow[t]{5}{*}{ Fluoxetina } & Amiodarona & $\uparrow$ risco de cardiotoxicidade & & $3(2,23)$ \\
\hline & & Levotiroxina & $\begin{array}{l}\text { Pode resultar em aumento das doses de } \\
\text { levotiroxina }\end{array}$ & & $3(2,23)$ \\
\hline & & *Amitriptilina & $\begin{array}{l}\uparrow \text { risco de toxicidade de antidepressivos } \\
\text { tricíclicos }\end{array}$ & & $3(2,23)$ \\
\hline & & *Imipramina & $\begin{array}{l}\uparrow \text { do risco de toxicidade de antidepressivos } \\
\text { tricíclicos, e síndrome da serotonina }\end{array}$ & & $2(1,49)$ \\
\hline & & Diazepam & $\uparrow$ concentrações séricas de diazepam & & $2(1,49)$ \\
\hline IMAO não seletivo & Imipramina & Resperidona & $\begin{array}{l}\text { O uso concomitante de ADT e antipsicóticospodem } \\
\text { resultar em um aumento do risco de cardiotoxicidade }\end{array}$ & & $2(2,23)$ \\
\hline \multirow[t]{2}{*}{ ISRS } & \multirow[t]{2}{*}{ Citalopram } & Piroxicam & Aumento do risco de hemorragia & & $2(2,23)$ \\
\hline & & *Imipramina & $\begin{array}{l}\text { Resultar em um aumento na biodisponibilidade e meia-vida } \\
\text { de desipramina, o metabolito principal da imipramina }\end{array}$ & & $1(0,74)$ \\
\hline \multirow[t]{2}{*}{ IMAO não seletivo } & \multirow[t]{2}{*}{ Amitriptilina } & *Venlafaxina & $\begin{array}{l}\text { Em um aumento do risco de } \\
\text { cardiotoxicidade }\end{array}$ & & $1(0.74)$ \\
\hline & & *Citalopram & $\begin{array}{l}\text { Pode resultar em um aumento do risco de } \\
\text { prolongamento do intervalo QT }\end{array}$ & & $1(0,74)$ \\
\hline ISRS & Paroxetina & Clobazam & $\begin{array}{l}\text { Pode resultar em aumento das concentrações } \\
\text { plasmáticas da paroxetina }\end{array}$ & & $1(0,74)$ \\
\hline IMAO não seletivo & Nortriptilina & *Citalopram & $\begin{array}{l}\text { Aumento do risco de prolongamento do } \\
\text { intervalo QT }\end{array}$ & & $1(0,74)$ \\
\hline \multirow[t]{2}{*}{ Outros } & Trazodona & *Amitriptilina & $\begin{array}{l}\text { pode resultar em um aumento do risco de prolongamento } \\
\text { do intervalo QT e síndrome da serotonina }\end{array}$ & & $1(0,74$ \\
\hline & Mirtazapina & Levotiroxina & $\begin{array}{l}\text { Aumento dos efeitos terapêuticos e tóxicos tanto } \\
\text { na levotiroxina e do antidepressivo }\end{array}$ & & $1(0,74)$ \\
\hline
\end{tabular}

$S$ maior; 4 moderada; (4) menor.

* interação medicamentosa em potencial envolvendo dois antidepressivos. 


\section{DISCUSSÃO}

No presente estudo, destaca-se que um quinto dos entrevistados fazia uso apenas de um medicamento antidepressivo e, portanto não suscetível à interação medicamentosa. Contudo, verificou-se que a maioria dos usuários apresentava potencial para desenvolver algum tipo de interação, de diferentes gravidades.

Um estudo realizado também com pacientes depressivos, cujo foco foi interações em geral, desenvolvido em um hospital público de São Paulo com 6.844 pacientes, encontrou 169 pacientes em uso de fármacos antidepressivos, sendo que 36 $(21,3 \%)$ apresentavam risco de interação medicamentosa. Entre antidepressivos e outros fármacos, 20 diferentes tipos de interações foram identificados, sendo quatro leves, 15 moderados e 1 grave $^{12}$. Verifica-se que no presente estudo foi encontrado um valor superior de interações em potencial n $(47,72 \%)$, mesmo que tenha sido desenvolvido com pacientes comunitários mostrando a necessidade de acompanhamento de usuários de antidepressivos. Já em outro estudo, desenvolvido em um hospital da rede pública de saúde em Campinas/SP, foram avaliadas as prescrições de 195 pacientes, sendo que 88,2\% apresentaram interações medicamentosas potenciais envolvendo diferentes grupos farmacológicos, destas 257 maiores, 516 moderadas e 122 menores $^{13}$. Em relação ao presente estudo, Mazzola et al..$^{13}$ encontrou um número mais elevado de interações, o que pode ser explicado pela alta complexidade do ambiente hospitalar, complexidade dos esquemas terapêuticos, a gravidade do estado clínico do paciente e pelo número elevado de medicamentos que o paciente necessita, estando mais predispostos a apresentar interações medicamentosas. Em contraponto, no presente estudo foram entrevistados em pacientes comunitários, que são propensos a utilizar um número menor de medicamentos.

Considerando que no presente estudo observou-se a prática de polifarmácia em vários pacientes, e essa tende a aumentar a incidência das interações medicamentosas, mostra-se a necessidade de avaliação das prescrições médicas. As das interações medicamentosas podem acarretar potencialização do efeito terapêutico, redução da eficácia, aparecimento de reações adversas, com distintas gravidades, ou, ainda, não causar nenhuma modificação no efeito desejado do medicamento ${ }^{14}$.

As interações medicamentosas são consideradas erros de medicação, com risco de dano em função da sua ocorrência, e torna-se, portanto, relevante identificar a natureza e determinantes destes erros, como forma de dirigir ações para a prevenção. As falhas no processo de utilização de medicamentos são consideradas importantes fatores contribuintes para a redução da segurança do paciente, sendo necessária a adoção de algumas condutas para prevenção e identificação dos fatores de risco, visando conhecer os mecanismos de ação farmacológica dos medicamentos em uso. Muitas interações dependem da dose, e nesse caso, a dose do medicamento pode ser reduzida para que o efeito do outro não seja inibido. Por outro lado uma maneira de evitar interações danosas é considerar a substituição de um dos fármacos por outro com propriedade similar e com menos risco de interação medicamentosa, evitando associação de fármacos com a mesma ação farmacológica quando não há prova suficiente que esta terá benéfico ao paciente ${ }^{15}$.

As interações medicamentosas em potencial com gravidade maior foram as mais prevalentes, resultado diferente dos outros estudos ${ }^{12,13}$, no qual prevaleceram as interações com gravidade moderada. Destaca-se a fluoxetina associada à amiodarona ou imipramina ou amitriptilina. Especificamente a interação fluoxetina e amiodarona pode resultar em um aumento do risco de cardiotoxicidade, com prolongamento do intervalo QT (atividade elétrica do ventrículo esquerdo), sendo que dependendo do grau de prolongamento do intervalo QT e do número de circunstâncias coexistente, podem ocorrer o desenvolvimento de torsades de pointes (arritmias ventriculares potencialmente letais) ${ }^{5,11}$. Alguns pacientes, no entanto, mesmo sem serem portadores de síndrome do QT longo genético clássico podem, quando fazem uso de alguns medicamentos, desenvolver um prolongamento do intervalo QT e também se exporem às arritmias ventriculares ${ }^{16}$. Estudos de prevalência estimam que 23 milhões de pessoas no mundo têm Insuficiência Cardíaca (IC) e que dois milhões de casos novos são diagnosticados anualmente. Há no Brasil cerca de dois milhões de pacientes com IC, sendo diagnosticados 240 mil casos novos por ano ${ }^{17}$, mostrando que devemos monitorar e ter cautela quanto ao uso de fármacos antiarrítmicos associados aos antidepressivos, para prevenir futuras interações medicamentosas e evitar eventos adversos indesejados. Foi verificado também que houve interações medicamentosas em potencial relacionadas a prescrição concomitante de antidepressivos de grupos diferentes, em especial os ISRS e IMAO não seletivo. De acordo com as normas de orientação terapêutica da Ordem dos Farmacêuticos de Portugal, faz-se uso destes fármacos em associação em depressão grave, e que não se obteve resposta terapêutica com o uso de apenas um grupo farmacológico ${ }^{18}$, sendo que no presente estudo 17 usuários faziam uso concomitante de dois ou mais antidepressivos.

Os protocolos de diagnóstico e tratamento da depressão recomendam que caso haja ausência de resposta ao tratamento proposto inicialmente deve-se tratar um episódio depressivo por pelo menos quatro semanas, antes de 
considerar a modificação de da terapia. Caso haja ausência na resposta ao tratamento em quatro semanas, deve-se verificar a dose e adesão ao tratamento, revisar também o diagnóstico do paciente, incluindo a possibilidade de presença de comorbidade psiquiátrica ou doença física que também deve receber tratamento. Se obtiver resposta parcial em quatro semanas continuar o tratamento, e na ausência de resposta em quatro semanas aumentar a dose, ou substituir por outra classe de antidepressivo, considerando a troca para IMAO em pacientes com sintomas atípicos (ganho de peso, hipersonia, hipersensibilidade e humor reativo a eventos externos). Na ausência de resposta a um segundo antidepressivo, pode-se adicionar um agente potencializador, ou a psicoterapia ou eletroconvulsoterapia ${ }^{2}$.

Foi observado que no presente estudo houve pacientes em uso de fluoxetina e imipramina, e este uso concomitante gera uma interação medicamentosa em potencial maior. Dados da literatura demonstram que a imipramina associado à fluoxetina pode aumentar de três a quatro vezes a concentração plasmática da imipramina ou desipramina, que é o metabólito principal da primeira ${ }^{8}$. Esses efeitos podem estar relacionados a capacidade da fluoxetina de inibir isoenzimas CYP2D6 e consequentemente, diminuir o clearance dos antidepressivos tricíclicos ${ }^{11}$, podendo gerar um quadro de intoxicação, relacionado com o fato da imipramina ser rapidamente absorvido por via oral, com elevada união a proteínas plasmáticas, tendo lento metabolismo hepático e eliminação renal, que pode demorar em vários dias ${ }^{19}$. De acordo com Fernandes et al. ${ }^{20}$ as principais manifestações clínicas por intoxicação destes fármacos são diminuição do nível de consciência, arritmias, excitação, coma, desconforto gastrintestinais e respiratório, podendo gerar insuficiência respiratória.

Por outro lado, a fluoxetina aumenta a concentração sérica de amitriptilina, e de seu metabólito ativo a nortriptilina, em aproximadamente 100 e 800\%, respectivamente ${ }^{5}$. Embora combinações de antidepressivos IMAO e ISRS sejam usadas com resultados positivos, os pacientes sob o uso de IMAO devem ser cuidadosamente monitorados quando um inibidor da recaptação de serotonina é iniciado, interrompido ou tenha sua dosagem modificada ${ }^{21}$ pelo fato da redução dos níveis de fluoxetina ocorrer de forma extremamente lenta em virtude da auto-inibição do seu metabolismo, a redução e/ ou interrupção desse composto tende a não ser eficaz diante de uma emergência clínica atribuiu-se morte à toxicidade crônica da amitriptilina causada pela fluoxetina ${ }^{8}$.

Observou-se associação de sertralina e citalopram, sendo esta a associação mais prevalente entre antidepressivos. Recomenda-se cautela e monitorização do aumento dos efeitos dos níveis de substrato do CYP (citalopram), quando for iniciada uma terapia com um inibidor de CYP (sertralina), pois este inibidor pode reduzir o metabolismo do substrato, diminuindo o clearence e aumentando a concentração sérica. $\mathrm{O}$ uso concomitante de dois ou mais fármacos deste grupo eleva o risco de aumento da atividade de serotonina a níveis tóxicos, causando a síndrome de serotoninérgica, que é caracterizada por pelo menos três dos seguintes sintomas: agitação, diaforese, diarréia, febre, hiper-reflexia, incoordenação, mioclonia, tremores e calafrios ${ }^{11}$. Sendo fármacos do mesmo grupo farmacológico, Os ISRS inibem de forma potente e seletiva a recaptação de serotonina, resultando em potencialização da neurotransmissão serotonérgica. Embora compartilhem o principal mecanismo de ação, os ISRS são estruturalmente distintos com diferenças no perfil farmacodinâmico e farmacocinético. A potência da inibição de recaptação da serotonina é variada, assim como a seletividade por noradrenalina e dopamina. A potência relativa da sertralina em inibir a recaptação de dopamina a diferencia farmacologicamente dos outros ISRS. A afinidade por neuroreceptores, tais como sigma, muscarínicos e 5-HT2c, também difere muito. Já o citalopram é uma mistura racêmica de diferente forma quiral que possuem perfil farmacodinâmico e farmacocinético variados. As concentrações plamáticas de sertralina e citalopram são proporcionais às doses administradas ${ }^{22}$.

Já nas interações medicamentosas em potencial de gravidade moderada mais frequentes foram representadas pela associação da fluoxetina a levotiroxina, assim como sertralina associado ao diazepam, que devem ser monitorados, considerando o aumento dos efeitos do substrato CYP (diazepam), quando for iniciada uma terapia com um inibidor CYP (sertralina), pois os inibidores podem reduzir o metabolismo do substratos dessa via. O clearance do substrato diminui e a concentração sérica aumenta ${ }^{11}$. A interação de gravidade menor foi representada pela associação de diazepam e fluoxetina, e nestas a fluoxetina pode diminuir o metabolismo do diazepam através de isoenzimas. Alguns estudos mostram que a interação entre fluoxetina e o diazepam pode provocar aumento nas concentrações séricas do benzodiazepínico, levando à prejuízos psicomotores e na atenção ${ }^{23}$. Isto ocorre porque o diazepam é substrato da isoenzima CYP (citocromo P450), sendo que muitos antidepressivos inibem esta isoforma ${ }^{11}$. Esta interação parece mais significativa com o uso contínuo do antidepressivo, portanto, é recomendável que o diazepam seja substituído por outro benzodiazepínico metabolizado por outras isoenzimas, como oxazepam ou temazepam ${ }^{24}$. Deve-se considerar que a interação medicamentosa em potencial é um risco real ao usuário, relacionado com a polifarmácia, e esta às morbidades dos usuários e os riscos de reações adversas aos medicamentos e potenciais interações 
medicamentosas, mostrando a necessidade da prática da atenção farmacêutica mais ativa ${ }^{25}$.

Embora nem todas as interações medicamentosas possam ser prevenidas, a difusão do conhecimento para os profissionais de saúde, quanto aos principais fatores de risco, mecanismos de ação das interações, associados à notificação das interações medicamentosas mais frequentes e relevantes na prática clínica, constituem um dos principais instrumentos de prevenção de danos aos pacientes. Esse conhecimento permitirá aos profissionais de saúde optarem por regimes terapêuticos e horários de administração de medicamentos mais seguros, conduzindo, assim, a assistência de qualidade e com maior segurança para o usuário ${ }^{26}$.

É importante ressaltar que existem interações medicamentosas desejáveis, que ajudam a reduzir efeitos adversos, prolongar a duração do efeito, impedir, aumentar a adesão ao tratamento, incrementar a eficácia ou permitir a redução de dose. Por sua vez, temos também as interações indesejáveis que são as que determinam redução do efeito ou resultado contrário ao esperado, aumento na incidência e na gama de efeitos adversos e o custo da terapia, sem incremento no benefício terapêutico. As interações que resultam em redução da atividade do medicamento e consequente perda da eficácia são difíceis de detectar e podem ser responsáveis pelo fracasso da terapia ou progressão da doença ${ }^{27}$.

Como limitação do estudo pode-se citar que interações com alimentos, medicamentos não prescritos e plantas medicinais não foram avaliados neste estudo. Outro aspecto a ser considerado é o fato de tratar-se de interações medicamentosas em potencial, e sem um estudo de acompanhamento, não pode-se afirmar se elas efetivamente ocorreram, já que não foram aplicados algoritmos para confirmação das mesmas. Mostrando a necessidade de outros estudos complementares avaliando esses aspectos, bem como a continuidade deste estudo na perspectiva de acompanhamento e intervenção.

Este estudo demonstra a ocorrência das interações medicamentosas em potencial na maioria dos usuários, sendo que a maioria ocorreu com os antidepressivos e foram de gravidade maior. Portanto, o uso concomitante de dois ou mais medicamentos, e principalmente a polifarmácia, pode expor o usuário a um risco real que pode ser prevenido com acompanhamento e orientações, a fim de otimizar o quadro clínico dos usuários e minimizar os riscos que podem advir das interações medicamentosas. Neste sentindo é de extrema importância que o farmacêutico esteja inserido em uma equipe multidisciplinar, pois através das intervenções farmacêuticas, e nas equipes este profissional contribui para a promoção do uso racional de medicamentos e para a segurança do paciente.

\section{REFERÊNCIAS}

1. Kaplan HI, Sadock BJ, Grebb JA. Compêndio de psiquiatria: ciências do comportamento e psiquiatria clínica. 9a ed. Porto Alegre: Artmed; 2007.

2. Fleck MPA, Berlim, MT, Lafer B, Sougey EB, Del Porto JA, Brasil MA, Juruena MF, Hetem LA. Revisão das diretrizes da Associação Médica Brasileira para o tratamento da depressão (versão integral). Rev Bras Psiquiatr. 2009;31(1):7-17. http://dx.doi.org/10.1590/ S1516-44462009000500003

3. Teng CT, Humes EC, Demetrio FN. Depressão e comorbidades clínicas. Rev. Psiq Clín. 2005;32(3):149-59. http://dx.doi.org/ 10.1590/S0101-60832005000300007

4. Organización Panamericana de la Salud. Tratamiento farmacológico de los trastornos mentales en la atención primaria de salud. Washington, D.C.: OPS; 2010.

5. Silva P. Farmacologia. 7a ed. Rio de Janeiro: Guanabara.Koogan; 2006.

6. Melgaco TB, Carrera JS, Nascimento DEB, Maia CSF. Polifarmácia e ocorrências de possíveis interações medicamentosas. Belém, PA: Universidade Federal do Pará; 2011.

7. Barros E, Barros HMT. Medicamentos na prática clínica. Porto Alegre: Artmed; 2010

8. Campigotto KF, Teixeira JJV, Cano FG, Sanches ACC, Cano MFF, Guimarães DSL. Detecção de risco de interações entre fármacos antidepressivos e associados prescritos a pacientes adultos. Rev Psiq Clín. 2009;35(1):1-5.

9. Pivatto Júnior F, Godoy DB, Pires DF, Pietrobon E, Rosa FT, Saraiva JS. Potenciais interações medicamentosas em prescrições de um hospital-escola de Porto Alegre. Rev AMRIGS. 2009;53(3):251-6.

10. Porto CC. Interação medicamentosa. Rio de Janeiro: Guanabara Koogan; 2011.

11. Bachmann KA, Lewis JD, Fuller MA, Bonfiglio MF. Interações medicamentosas. Barueri: Manole; 2006.

12. Miyasaka LS, Atallah NA. Risco de interação de drogas: combinações de uso de antidepressivos e outras drogas. Rev Saud Pub. 2003;37:212-15. http://dx.doi.org/10.1590/S003489102003000200008

13. Mazzola PG, Rodrigues AT, Cruz AA, Marialva M, Granja S, Battaglini SCM. Perfil e manejo de interações medicamentosas potenciais teóricas em prescrições de UTI. Rev Bras Farm Hosp Serv Saúde. 2011;2(2):15-9.

14. Secoli SR. Drugs interactions: fundamental aspects for clinical practice nursing. Rev Esc Enferm USP. 2001;35(1):28-34. http:// dx.doi.org/10.1590/S0080-62342001000100005

15. Ministério da Saúde (BR). Secretaria de Ciência, tecnologia e Insumos Estratégicos. Departamento de Assistência Farmacêutica e Insumos Estratégicos. Formulário terapêutico nacional 2010: Rename 2010. 2ª ed. Brasília: Ministério da Saúde; 2011.

16. Barcelos AM, Teixeira MA, Maia MC, Camanho LEM, Asumpção OQ. Síndrome do QT longo e torsades de pointes pós-parto. Arq Bras Cardiol. 2009;93(4):58-9. http://dx.doi.org/10.1590/S0066782X2009001000022

17. Nogueira PR, Rassi S, Corrêa KS. Perfil epidemiológico, clínico e terapêutico da insuficiência cardíaca em hospital terciário. Arq Bras Cardiol, 2010;93(4):58-9. http://dx.doi.org/10.1590/s0066$782 \times 2010005000102$ 
18. Caramona M, Vitória I, Teixeira M, Alcobia A, Almeida P, Horta R, Reis, L. Normas de orientação terapêutica. Lisboa: Ordem dos Farmacêuticos; 2011.

19. Ministério da Saúde (BR). Protocolo de segurança na prescrição, uso e administração de medicamentos. Brasília: Ministério da Saúde; 2014.

20. Fernandes G, Palvo F, Pinton FA, Dourado DAN, Mendes CAC. Impacto das intoxicações por antidepressivos tricíclicos comparados aos depressores do sistema nervoso central. Arq Ciênc Saúde. 2006;13:61-5.

21. Greghl CM, Moraes DSC, Zanluchi SL, Barbosa NRA, Valle R, Lopes SRS. Interações medicamentosas envolvendo os neuropsicofármacos padronizados no HURNP. In: Anais da V Jornada de Farmácia e Análises Clínicas de Londrina; 2001. p. 72.

22. Paulin LFRS, Reis EF, Rodrigues EF. Síndrome de descontinuação dos antidepressivos. RBM Rev Bras Med. 2008;65(10):326-30.

23. Marcolin MA, Cantarelli MG, Garcia Junior M. Interações farmacológicas entre medicações clínicas e psiquiátricas. Rev Psiq Clín. 2004; 31(2):70-81. http://dx.doi.org/10.1590/s0101-60832004000200003
24. Santos HC, Ribeiro RR, Ferrarini M, Fernandes JPS. Possíveis interações medicamentosas com psicotrópicos encontradas em pacientes da Zona Leste de São Paulo. Rev Cienc Farm Básica. 2009;30(3):285-89.

25. Furini AAC, Lima TAM, Faitarone NC, Verona JP, Silva LAM, Santos SS. Atenção farmacêutica nas interações medicamentosas e indicadores de prescrição em unidade básica de saúde. Arq Ciênc Saúde. 2014;21(2)99-106.

26. Lima REF, Cassiane SHDB. Interações medicamentosas potenciais de unidade de terapia intensiva de um hospital universitário. Rev Latino-Am Enfermagem. 2009;17(2):81-7.

27. Istilli PT, Miasso AI, Padovan CM, Crippa JA, Tirapelli CR. Antidepressivos: uso e conhecimento entre estudantes de enfermagem. Rev Latino-Am Enfermagem. 2010;18(3):131-39.

28. MICROMEDEX ${ }^{\circledR}$ Healthcare Series [Internet database]. Greenwood Village, CO: Thomson Reuters; 2015 [updated periodically]. Available from: http://www.micromedexsolutions.com/micromedex 2/librarian 